\title{
OSCILLATORY CONVECTION IN STRONG MAGNETIC FIELDS AND ORIGIN OF ACTIVE REGIONS
}

\author{
S. I. SYROVATSKY \\ (Physical Institute of Lebedev, \\ Moscow, U.S.S.R.)
}

\author{
and
}

Y.D. ZHUGZHDA

(Institute of Earth Magnetism, Ionosphere and Propagation

of Radio-Waves,

Academy of Sciences, Moscow, U.S.S.R.)

\begin{abstract}
A BSTRACT
The convection in a compressible inhomogeneous conducting fluid in the presence of a vertical uniform magnetic field has been studied. It is shown that a new mode of oscillatory convection occurs, which exists in arbitrarily strong magnetic fields. The convective cells are stretched along the magnetic field, their horizontal dimensions are determined by radiative cooling. Criteria for convective instability in a polytropic atmosphere are obtained for various boundary conditions in the case when the Alfvén velocity is higher compared with the velocity of sound.

The role of oscillatory convection in the origin of sunspots and active regions is discussed.
\end{abstract}

A number of problems of solar physics are connected with the effect of the magnetic field on the convective transfer of heat. The main question is whether the magnetic field suppresses the convection in vertical field or the convective heat transport has place in the strong magnetic field too, because of an existence of some other type of convective motions. Both theoretical investigations of sunspots structure (Chitre, 1963; Deinzer, 1965), as well as observations of granulation in umbra (Danielson, 1964; Bray and Loughhead, 1964) show that the convection is not suppressed in spots.

The fact that the usually considered circulatory convection is suppressed in sunspots can be verified by using the criterion of stability, obtained in the most general form by Gough and Tayler (1966). It follows then that non-circulatory convection must occur in the strong magnetic field of spots.

Oscillatory convection in sunspots was investigated by Danielson (1965) and Danielson and Savage (1968) in the frame of the theory of convection in an incompressible fluid (Chandrasekhar, 1961). Results of Chandrasekhar's theory can be used for astrophysical objects only if the unstable layer is thin and can be approximated by the layer with constant density. But the solar convective zone is thick and we need therefore a theory of convection in non-uniform, compressible atmosphere.

The aim of the present investigation is to search for convection mode, which is not suppressed by any magnetic field. This problem was initiated in the paper of Syrovatskii and Zhugzhda (1967), and the properties of this convection are investigated by Zhugzhda in more detail (1968).

Kiepenheuer (ed.), Structure and Development of Solar Active Regions, 127-130. O I.A.U. 
We consider convection in a compressible inhomogeneous atmosphere in the presence of a vertical magnetic field. The general linearized equations of magnetohydrodynamics for a polytropic atmosphere can be simplified in the following way:

It is evident that a strong vertical magnetic field will not prevent oscillatory motion along lines of force. In a uniform medium the motion of this type corresponds to a slow magneto-acoustic wave, which propagates almost transversally to the field. We assume that oscillatory motions along the field in non-uniform medium also have the properties of a slow magneto-acoustic wave. Besides the equations can be simplified on the condition that the Alfvén velocity is large as compared to the acoustic one and convective cells are stretched along force lines. It appears that the obtained equation describes acoustic oscillations of gas along magnetic force lines in a non-uniform medium. The following equation was obtained:

$$
z^{2} \frac{\mathrm{d}^{2} w}{\mathrm{~d} z^{2}}+\left(n+1-\begin{array}{c}
q \\
\gamma \sigma+q
\end{array}\right) z \frac{\mathrm{d} w}{\mathrm{~d} z}-\left(\begin{array}{cc}
\sigma^{2}(n+1)(\sigma+q) & n q \\
g(\gamma \sigma+q) & z+\ldots+q
\end{array}\right) w=0,
$$

where $w=$ vertical component of velocity, $z=$ depth in atmosphere, $n=(\mu g / R \beta)-1$ is polytropic index, $\mu=$ molecular weight, $g=$ acceleration due to gravity, $R=$ gas constant, $\beta=$ constant temperature gradient $\left(T_{0}=\beta z\right), q=$ inverse time for radiative cooling (see Spiegel, 1957), $\sigma=\mathrm{i} \omega+\varkappa=$ complex frequency, $\omega=$ frequency of convective oscillation, $\varkappa=$ increment of linear theory, and $\gamma=$ ratio of specific heats.

The equation solution is expressed by Bessel's functions.

This oscillatory motion in the strong magnetic field is unaffected by field strength. If heat transfer does not occur between adjacent cells $(q=0)$, the oscillatory motions are adiabatic and undamped. Oscillatory convective motions grow in time provided there is heat transfer between adjacent convective cells $(q \neq 0)$. If $q$ does not depend

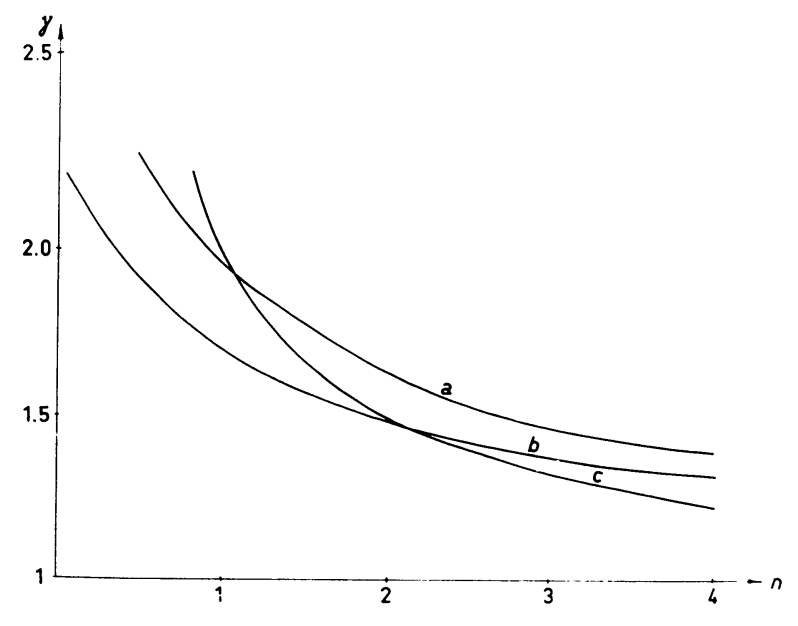

FIG. 1 . 
on the depth of cells ( $q=$ const) the position of instability-region boundary for fixed value of $q$ depends only on ratio of specific heats and polytropic index $n$.

The instability criterion $(\varkappa=0)$ depends in general on the inverse time for radiative cooling to oscillatory frequency ratio. Consequently, in the case of optically thick cells the instability criterion depends on the cell size. There are two limiting cases. If convective cells are thick and the inverse time for radiative cooling is small compared with angular frequency $(q \ll \omega)$ the convective oscillations are quasi-adiabatic. If the convective cells are thin and the inverse time for radiative cooling is large compared with angular frequency, the convective oscillations are quasi-isothermal $(q \gg \omega)$.

The slide (Figure 1) shows criteria of instability (for rigid boundary conditions) for these limiting cases in the $(\gamma, n)$ diagram. Let us consider an atmosphere which consists of gas with definite molecular weight and definite ratio of specific heats. If the temperature gradient increases, we move to the left along the line parallel to the abscissa axis. When we cross the curve labelled $a$, the oscillatory convection with very thin cells arises. When we cross the curve labelled $b$, the oscillatory convection with very thick cells arises. Instability boundaries for intermediate size of cells are placed between these curves. What size of cells must be realized? It can be shown that viscosity is not of importance here. The maximum increment corresponds to such size of cells at which the inverse time for radiative cooling appears to be of order of oscillation frequency. Simultaneously this size of cells corresponds to maximum heat flux. For this case the instability boundary is located approximately halfway between the curves labelled $a$ and $b$.

We have considered the case when the inverse time of cooling is unaffected by temperature and gas pressure. In this case the solution of the obtained equation is expressed by analytical functions. It is given also another method of obtaining criteria. This method consists of the energy-balance calculation. The criterion for atmosphere with an arbitrary dependence of inverse cooling time on temperature can be obtained by this method.

The criterion is strongly dependent on boundary conditions. With this in mind, the effect of the motion penetration into stable layers on instability criteria was investigated. This investigation permits to obtain criteria of instability for real astrophysical conditions. These criteria are affected by the structure of layers lying above and below the unstable layer. The main result is that the obtained criteria of instability are the same or more strict in comparison with the criteria demonstrated in Figure 1.

The heat transport is an important property of oscillatory convection. The flux of heat is equal to

$$
I={ }_{2 \omega}^{\gamma \lambda K_{\perp}^{2}} T^{\prime} v \xi
$$

where $i=$ thermal conductivity, $K_{\perp}^{2}=$ square of horizontal wave vector, $T^{\prime}$ and 
$v=$ amplitudes of temperature and velocity oscillations, $\xi=$ factor which depends on phase differences of these oscillations.

There is also non-thermal flux of energy. This non-thermal flux transports the energy within unstable layers and out into adjacent stable layers, and thus changes an initial polytropic atmosphere.

The curve (Figure 1) labelled $c$ is Schwarzschild's criterion for non-conducting fluid. The origin of sunspots may be treated considering the fact that in atmosphere with ratio of specific heats around $5 / 3$, the oscillatory convection arises at a larger temperature gradient compared with the adiabatic one. Consequently, the temperature gradient in sunspots is larger compared with the temperature gradient in convection zone. More careful consideration of spot origin was undertaken, which takes into account the penetration of motions into stable layers; some other factors were also taken into account. It is interesting that in the atmosphere with ratio of specific heats around unity, the oscillatory convection arises at a smaller temperature gradient compared with the adiabatic one. This may be a cause of a hotter region appearance. But the complete consideration of this problem demands a solution of the same problem for the more general case of intermediate values of magnetic-field strength, which has not yet been done.

\section{References}

Bray, R., Loughhead, R. (1964) Sunspots, Chapman and Hall Ltd., London.

Chandrasekhar, S. (1961) Hydrodynamic and Hydromagnetic Stability.

Chitre, S. M. (1963) Mon. Not. R. astr. Soc., 126, 431.

Danielson, R.E. (1964) Astrophys. J., 139, 45.

Danielson, R.E. (1965) in I.A.U. Symposium No. 22, Ed. by R. Lüst, North-Holland Publ. Co., Amsterdam, p. 314.

Danielson, R.E., Savage, B.D. (1968) the present volume, p. 112.

Deinzer, W. (1965) Astrophys. J., 141, 548.

Gough, D.O., Tayler, R.J. (1966) Mon. Not. R. astr. Soc., 133, 85.

Spiegel, E. A. (1957) Astrophys. J., 126, 202.

Syrovatskii, S. I., Zhugzhda, Y.D. (1967) Astr. Zu., 44, 1180.

Zhugzhda, Y.D. (1968) Astr. Zu., in press. 